\title{
Présentation antigénique par les cellules dendritiques
}

\section{Sebastian Amigorena}

\section{ADRESSE}

S. Amigorena: directeur de recherche au Cnrs. Section de recherche, Inserm U. 520, Institut Curie, 12, rue Lhomond, 75005 Paris, France.

$m / s n^{\circ} 8-9$, vol. 15, août-septembre 99

Pour entraîner une réponse immune, l'antigène stimulant doit être présenté au lymphocyte $\mathrm{T} \mathrm{CD8}^{+}$(réponse cytolytique) ou $\mathrm{CD}^{+}$(réponse auxiliaire) par une molécule du CMH, respectivement de classe I ou II. Parmi les cellules présentatrices de l'antigène, les cellules dendritiques sont les plus performantes : elles ont la capacité non seulement d'activer des lymphocytes $T$ naifs, mais aussi d'induire une réponse humorale et cytolytique par leur présentation d'antigènes par les molécules de classe I ou II du CMH. Les étapes du catabolisme de l'antigène sont strictement corrélées aux stades de la maturation des cellules dendritiques: seules les cellules dendritiques immatures, concentrées dans les tissus phériphériques, peuvent phagocyter et provoquer l'endocytose des antigènes solubles et particulaires, mais elles ne peuvent pas, à ce stade, les présenter aux lymphocytes $\mathbf{T}$ car les molécules du CMH sont retenues dans les lysosomes. En revanche, le processus de maturation de ces cellules, contrôlé par des signaux inflammatoires et par le couple $\mathrm{CD} 40-\mathrm{L} / \mathrm{CD} 40$, s'accompagne de changements morphologiques, de la relocalisation des molécules de classe II du CMH à la membrane, de l'expression de molécules de co-stimulation lymphocytaire, et surtout de la migration des cellules dendritiques des tissus périphériques vers les ganglions et la rate. Dans ces organes, la cellule dendritique mûre est apte à présenter aux lymphocytes $\mathrm{CD4}^{+}$l'antigène protéolysé en peptides et complexé aux molécules du CMH-II.

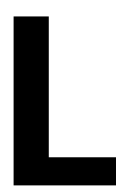

a mise en route des réponses immunitaires repose sur un événement de reconnaissance moléculaire complexe: la liaison entre les récepteurs des antigènes exprimés par les lymphocytes T (TCR), d'une part, et des complexes formés par des peptides engendrés par dégradation protéolytique des antigènes et les molécules du complexe majeur d'histocompatibilité $(\mathrm{CMH})$, d'autre part [1]. Il existe deux classes de molécules du CMH. Les molécules de classe I (HLA A, B et $\mathrm{C}$ chez l'homme et $\mathrm{H} 2-\mathrm{K}$ et $\mathrm{D}$, chez la souris) et les molécules de classe II (HLA-DR, DP et DQ chez l'homme, I-A et I-E, chez la souris). Les molécules de classe I présentent des peptides antigéniques aux lymphocytes cytotoxiques (exprimant les co-récepteurs CD8), alors que les molécules de classe II présentent des peptides aux lymphocytes $\mathrm{T}$ auxiliaires (ou $\mathrm{CD} 4^{+}$). 
De nombreux travaux menés depuis une dizaine d'années ont montré que les molécules de classe I présentent principalement des peptides dérivés de protéines endogènes (synthétisés par les cellules elles-mêmes), souvent localisés dans le cytosol [2]. Les molécules de classe II, au contraire, présentent aussi bien des protéines endogènes membranaires ou sécrétées que des protéines exogènes entrées dans les cellules présentatrices par internalisation. Cette spécificité de présentation repose, au moins en partie, sur le mécanisme même de production des peptides antigéniques et leur site intracellulaire d'association aux molécules du CMH. En effet, les molécules de classe I s'associent dans le réticulum endoplasmique, aussitôt après leur synthèse, à des peptides produits dans le cytosol et transloqués dans le réticulum endoplasmique par des pompes spécifiques appelées TAP1/2. Les molécules de classe II, incapables de lier des peptides dans le réticulum endoplasmique en raison de leur association avec la chaîne invariante (Ii), sont transportées vers la voie endocytique où elles s'associent à des peptides antigéniques. In fine, la distinction entre antigènes exogènes et endogènes repose donc sur la compartimentalisation intracellulaire, c'est-à-dire sur le fait que le compartiment cytosolique est inaccessible depuis l'extérieur des cellules ou depuis le milieu endocytique.

Ce modèle est séduisant, car il explique pourquoi les lymphocytes $\mathrm{T}$ cytotoxiques détruisent les cellules infectées (dans le cas d'infections virales, en tout cas), même celles qui ont pu internaliser des agents infectieux inactifs ou des antigènes sécrétés par ces organismes, et non les cellules saines. La plus faible sélectivité de présentation par les molécules de classe II du CMH, qui présentent des peptides dérivés d'antigènes exogènes ou endogènes (en tout cas si ceux-ci sont des protéines membranaires ou sécrétées), reflète probablement la nécessité de l'intervention des lymphocytes $\mathrm{T}$ auxiliaires $\mathrm{CD} 4^{+}$ pour les réponses de type humoral et cytotoxique.

Cependant, il apparaît clairement aujourd'hui que ce modèle est trop réducteur, probablement en raison

la présentation antigénique par des cellules ayant un rôle d'effecteur dans la réponse immunitaire. En effet, la présentation par les molécules de classe II et de classe I a été principalement étudiée, respectivement sur des lymphocytes B et sur des cellules non hématopoiétiques. Or, au niveau du déclenchement des réponses immunes, la situation semble très différente. En effet, les cellules infectées dans un tissu ou les cellules tumorales ne sont pas capables d'activer directement les lymphocytes $\mathrm{T}$ cytotoxiques naïs (n'ayant encore jamais rencontré leur antigène spécifique) et donc de déclencher des réponses immunes. L'activation de lymphocytes $\mathrm{T}$ naifs nécessite l'expression de molécules de co-stimulation (comme CD80 ou CD86), qui ne sont pas présentes sur les cellules non hématopoiétiques. De plus, le développement de réponses immunitaires - cellulaires et humorales - implique des lymphocytes $\mathrm{T}$ auxiliaires et donc la présentation d'antigènes par les molécules de classe II du CMH qui, en règle générale, ne sont pas exprimées par les cellules périphériques ou tumorales.

Les antigènes exprimés par les cellules infectées ou tumorales doivent donc être présentés aux lymphocytes $\mathrm{T}$ par des cellules présentatrices dites "professionnelles", exprimant, outre des molécules de classe I, des molécules de co-stimulation et des molécules de classe II du CMH. Ces cellules doivent donc être capables d'acquérir des antigènes externes (de cellules infectées ou de cellules tumorales) et de les présenter en association avec les molécules de classe I du CMH aux lymphocytes T cytotoxiques [3]. Ce phénomène, appelé cross priming ou cross presentation (présentation croisée), initialement décrit par M. Bevan en 1976 [4], était récemment encore mal compris. Au cours des cinq dernières années, de nombreux travaux, in vivo et in vitro, suggèrent fortement qu'une famille de cellules hématopoiétiques, appelées cellules dendritiques, est à l'origine des réponses immunitaires, et notamment à la base du phénomène de cross priming [3]. Les cellules dendritiques constituent une famille cellulaire hétérogène d'origine médullaire [5]. Ces cellules ont la caractéristique fonctionnelle commune d'être les seules capables d'activer des lymphocytes $\mathrm{T}$ naïfs et de déclencher des réponses immunitaires, aussi bien humorales que cytotoxiques. Pour ce faire, les cellules dendritiques ont développé des caractéristiques uniques de maturation fonctionnelle et de présentation antigénique par les molécules du CMH.

\section{Maturation des cellules dendritiques}

Les cellules dendritiques peuvent se trouver sous deux formes distinctes de maturation (figure 1). Dans les tissus périphériques, elles sont principalement dans un état immature, caractérisé par un ensemble de molécules de surface et de propriétés fonctionnelles $[5,6]$. Les cellules dendritiques immatures expriment faiblement des molécules du CMH et de co-stimulation lymphocytaire (CD40, CD80, CD86). Toutes ces molécules sont synthétisées à des concentrations relativement faibles et s'accumulent dans des compartiments intracellulaires d'origine endocytique. Cela rend les cellules dendritiques immatures inefficaces pour stimuler des lymphocytes $\mathrm{T}$ (figure 1). En revanche, les cellules dendritiques immatures ont une forte capacité d'endocytose, de macropinocytose (qui est constitutive dans les cellules dendritiques immatures, contrairement aux autres types cellulaires étudiés à ce jour) et de phagocytose, ce qui leur permet d'ingérer des antigènes de manière très efficace [7]. Au moins deux récepteurs spécifiques sont impliqués dans l'endocytose d'antigènes par les cellules dendritiques immatures, les récepteurs du mannose, qui permettent l'endocytose efficace d'antigènes glycosylés [8], et les récepteurs Fc $(\mathrm{RFc})$ qui augmentent l'endocytose de complexes antigène-anticorps (complexes immuns) [8].

Différents signaux déclenchent la différenciation des cellules dendritiques immatures en cellules dendritiques mûres [2]. Des composants des parois bactériennes (comme le lipopolysaccharide, LPS) ou l'infection par des virus (comme l'influenza), d'une part, et des cytokines de l'inflammation (comme le TNF $\alpha$ ou 


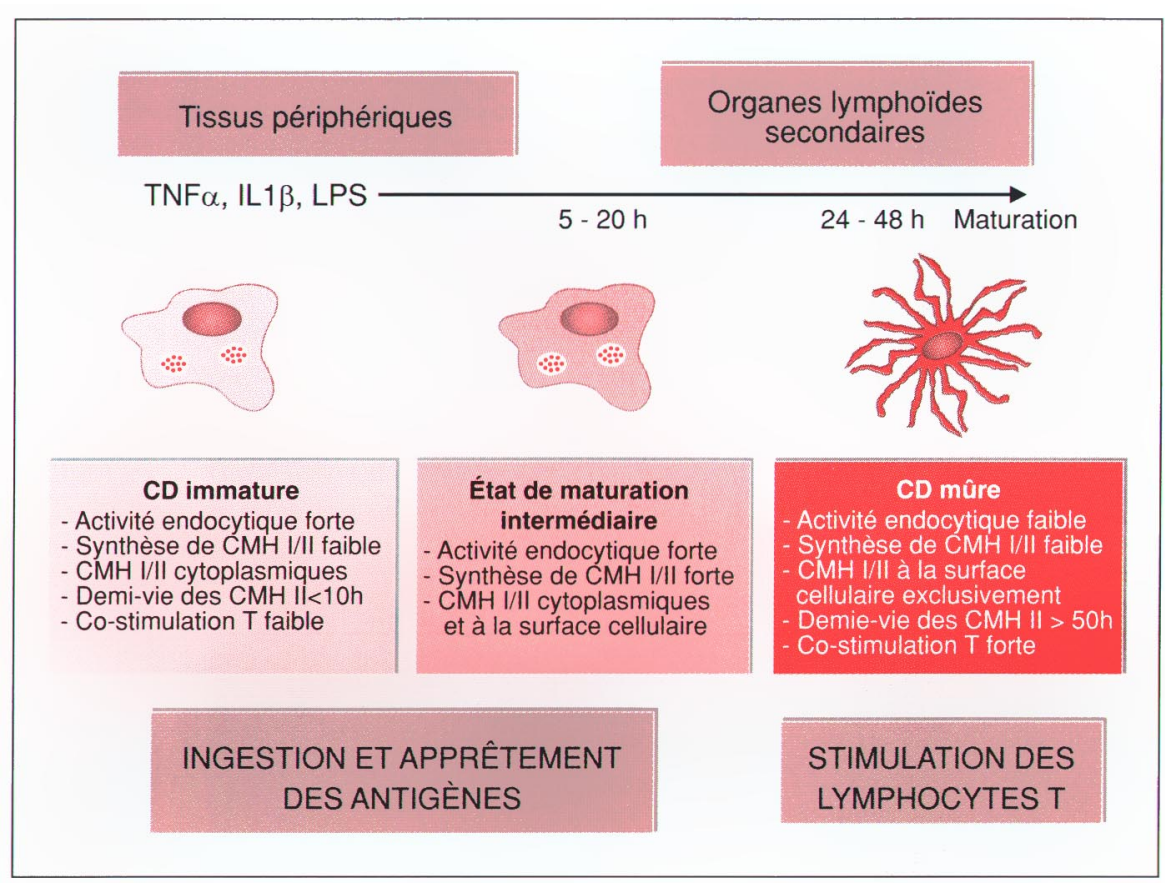

Figure 1. États de maturation fonctionnels des cellules dendritiques. Les cellules dendritiques sont activées par différentes cytokines (IL-1, TNF $\alpha$ ) ou des composants des parois bactériennes (lipopolysaccharides, LPS). La maturation des cellules dendritiques comprend deux étapes distinctes: un état de maturation intermédiaire, dans lequel les cellules dendritiques sont particulièrement efficaces pour ingérer et apprêter les antigènes, et un état de maturation finale, dans lequel elles sont compétentes pour l'activation des lymphocytes $T$. CD: cellule dendritique; TNF $\alpha$ : tumor necrosis factor $\alpha ;$ IL-1 $\beta$ : interleukine-1 $\beta$.

l'IL-1), d'autre part, provoquent la maturation des cellules dendritiques (figure 1). De plus, les lymphocytes T eux-mêmes, grâce à l'interaction du ligand de CD40 - présent sur les lymphocytes $\mathrm{T}$ - et de CD40 - présent sur les cellules dendritiques - participent à la maturation finale des cellules dendritiques, nécessaire à la stimulation de lymphocytes $\mathrm{T}$ cytotoxiques [2] (figure 2). Une série de travaux récents a montré que la même cellule dendritique doit nécessairement recevoir un signal des lymphocytes $\mathrm{T}$ auxiliaires (grâce au système $\mathrm{CD} 40 /$ ligand de $\mathrm{CD} 40$ ) pour devenir compétente pour la stimulation de lymphocytes cytotoxiques [9, 10]. Enfin, nous avons montré récemment que les complexes immuns aussi, en se liant aux récepteurs de la région $\mathrm{Fc}$ des immunoglobulines $\mathrm{G}(\mathrm{RF} c \gamma)$, provoquent la maturation des cellules dendritiques murines [11]. L'induction de la maturation des cellules dendritiques nécessite la chaîne $\gamma$ associée aux $\mathrm{RFc}$, puisque des complexes immuns n'induisent pas la maturation des cel-

$\mathrm{m} / \mathrm{s} n^{\circ} 8-9$, vol. 15 , août-septembre 99 tissus périphériques pour migrer vers les organes lymphoïdes secondaires, ganglions et rate, principalement.

Ce modèle de maturation des cellules dendritiques, proposé à partir de nombreux travaux effectués in vivo et in vitro, rend compte de la capacité exceptionnelle des cellules dendritiques de mettre en route des réponses immunitaires: les cellules dendritiques dispersées dans les tissus périphériques ingèrent les antigènes solubles et particulaires, de manière extrêmement efficace aussi bien par phase fluide que par des récepteurs spécifiques. Ces antigènes sont digérés ou stockés dans des compartiments endocytiques particuliers; les peptides antigéniques sont éventuellement engendrés et s'associent aux molécules du $\mathrm{CMH}$, mais les complexes CMH-peptides sont retenus à l'intérieur des cellules. L'absence d'expression de molécules de co-stimulation à la surface des cellules dendritiques immatures rend, de toute façon, la stimulation de lymphocytes $\mathrm{T}$ inefficace. Pour obtenir une stimulation lymphocytaire $\mathrm{T}$, il faut un «deuxième signal », signal de maturation donné soit directement par les agents pathogènes euxmêmes, soit indirectement par certaines des cytokines produites au cours des phénomènes inflammatoires. Ces signaux de maturation des cellules dendritiques, en augmentant l'expression des molécules du CMH et de co-stimulation lymphocytaire à la surface des cellules dendritiques et leur migration vers les organes lymphoïdes secondaires, permettent la mise en route des réponses immunitaires. Ainsi, en l'absence d'un signal de maturation, l'ingestion d'antigènes (comme les antigènes du soi) ne déclenche pas de réponse immunitaire. Ce mécanisme pourrait contribuer à limiter les réponses auto-immunes.

Dans le cadre de ce modèle de maturation des cellules dendritiques, la mise au point de systèmes de culture in vitro des cellules dendritiques humaines et murines, a permis, au cours des cinq dernières années, d'analyser en détail les mécanismes de présentation antigénique par les molécules de classe I et II du CMH au cours de la maturation des cellules dendritiques. 


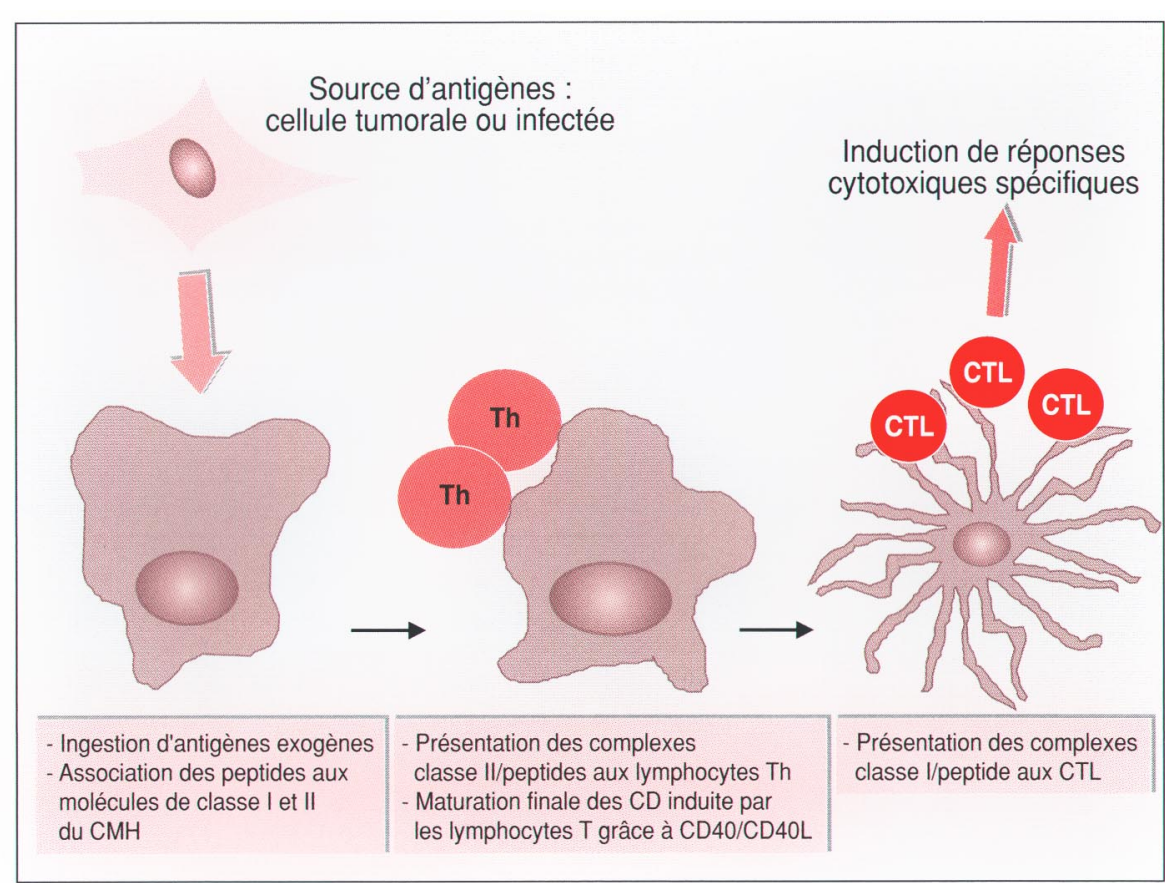

Figure 2. Cellules dendritiques et activation des lymphocytes $T$ cytotoxiques. Après avoir ingéré des antigènes, les cellules dendritiques doivent interagir avec des lymphocytes $T$ auxiliaires (CD4) qui, par stimulation grâce au CD40L, permettent aux cellules dendritiques d'activer efficacement les lymphocytes cytotoxiques. Le développement d'une réponse CTL (lymphocytes $T$ cytotoxiques) nécessite donc la présentation de peptides antigéniques par les molécules du CMH de classe I et II. CD: cellules dendritiques.

\section{Présentation d'antigènes par les molécules de classe II du CMH}

Les mécanismes fondamentaux reliant la présentation antigénique par les molécules de classe II et la maturation ont été analysés en détail, dans des cellules dendritiques humaines et murines, principalement par les équipes d'A. Lanzavecchia et d'I. Mellman, respectivement. Les résultats de ces deux séries de travaux sont très semblables, mais diffèrent dans certains aspects, qui cependant n'affectent pas les conclusions biologiques fondamentales. Ces différences pourraient être dues à des différences d'origine ou d'espèce des cellules dendritiques. Il est clair, néanmoins, que ces études associent au rôle central des cellules dendritiques dans la mise en route des réponses immunitaires, une série unique de modifications fonctionnelles de présentation antigénique au cours de la maturation.

Ces études ont été possibles grâce au développement par différentes immatures ingèrent et dégradent les antigènes, dans les endosomes et les lysosomes, de manière extrêmement efficace [7]. Dans les cellules dendritiques immatures, la majorité des molécules de classe II du CMH résident dans ces compartiments endocytiques, ce qui optimise l'association des peptides issus de la dégradation antigénique aux molécules de classe II du CMH [8]. Les mécanismes responsables de la rétention lysosomale des molécules de classe II semblent différents dans les cellules dendritiques humaines et murines. Chez l'homme, l'équipe d'A. Lanzavecchia a montré que la rétention lysosomale des molécules de classe II est probablement due à un taux d'internalisation constitutif très élevé, ce qui maintiendrait à l'état d'équilibre une majorité des molécules de classe II dans les endosomes et les lysosomes [15]. Dans ces cellules, les molécules de classe II du CMH néosynthétisées (et donc associées à la chaîne Ii) sont en majorité transportées directement vers la surface cellulaire, puis internalisées [16]. La chaîne Ii est ensuite dégradée, avant le chargement des peptides et le recyclage des molécules de classe II vers la membrane plasmique. Dans les cellules dendritiques murines, les mécanismes de rétention lysosomale semblent très différents [17]. En effet, les molécules de classe II néosynthétisées sont transportées directement vers la voie endocytique, où la dégradation de la chaîne Ii est démarrée, et se poursuit jusqu'à formation d'un fragment de 10 kDa (Ii-p10), composé d'une partie de la région luminale de la chaîne Ii (comprenant la région CLIP), ainsi que des régions transmembranaire et cytoplasmique. Dans d'autres types cellulaires, comme les lymphocytes B, Ii-p10 est très rapidement dégradé par la cathepsine S. Dans les cellules dendritiques immatures, un inhibiteur de la cathepsine $\mathrm{S}$, la cystatine $\mathrm{C}$, empêche la dégradation de Ii-p10, provoquant la rétention lysosomale des molécules de classe II néosynthétisées [18]. Lors de la maturation des cellules dendritiques, l'activité inhibitrice de la cystatine $\mathrm{C}$ dans les lysosomes disparaît et Ii-p10 est dégradé, permettant ainsi le chargement d'un peptide antigénique et le transport des molécules de classe II vers la surface cellulaire. 
Par ces deux moyens - taux d'internalisation élevé ou rétention - les molécules de classe II du CMH s'accumulent dans la voie endocytique des cellules dendritiques immatures, ce qui rend la présentation d'antigènes aux lymphocytes $\mathrm{T}$ inefficace. Une autre conséquence importante de cette localisation lysosomale des molécules de classe II du CMH dans les cellules dendritiques immatures est que leur durée de vie est très courte (figure 1). En effet, les molécules de classe II ont une durée de vie de 5-10 heures dans les cellules dendritiques immatures humaines ou murines, alors qu'elles ont une demivie beaucoup plus longue dans les lymphocytes B [17, 19]. Par conséquent, même si des peptides antigéniques sont chargés sur les molécules de classe II, ces complexes sont rapidement dégradés, ce qui diminue la probabilité pour les cellules dendritiques immatures de stimuler des lymphocytes T. Lors de la maturation des cellules dendritiques, la relocalisation des molécules de classe II à la membrane cytoplasmique provoque une remarquable augmentation de leur durée de vie (elle passe de 10 à 50-100 heures). La durée de vie des complexes molécules de classe IIpeptides engendrés par les cellules dendritiques immatures sera donc prolongée si un signal de maturation accompagne la rencontre des antigènes $[17,19]$.

Par conséquent, un ensemble coordonné de modifications fonctionnelles, qui accompagnent la maturation, rend compte de la capacité, spécifique et unique, des cellules dendritiques de mettre en route des réponses immunitaires (figure 1). L'augmentation du taux de synthèse des molécules de classe II se fait très rapidement (dans les deux heures suivant la stimulation), et avant la diminution de l'activité endocytique [19]. Parallèlement, les molécules de classe II passent de compartiments endocytiques de type lysosomal à des compartiments d'origine endosomique (vésicules de classe II, CIIV) [17]. Il semble donc que, pendant les premières $18-24$ heures suivant l'activation, les cellules dendritiques entrent dans un état de maturation intermédiaire (figure 1), toutes les conditions étant réunies pour optimiser l'ingestion des anti- gènes, la production protéolytique de peptides et l'association de ces peptides aux molécules de classe II du CMH.

Après cette période, les cellules dendritiques rentrent dans une phase réfractaire, dans laquelle la diminution de l'activité endocytique et du taux de synthèse des molécules de classe II, ainsi que la redistribution des molécules de classe II vers la membrane plasmique font que, même si elles rencontrent des antigènes, il n'y aura pas de présentation antigénique. En revanche, la stabilisation des molécules de classe II préexistantes a pour conséquence la mise en place d'une "mémoire antigénique»qui permet aux épitopes engendrés dans les cellules dendritiques immatures ou immédiatement après l'activation, de perdurer. Cela est vraisemblablement capital pour permettre aux cellules dendritiques de stimuler les lymphocytes $\mathrm{T}$ après avoir migré vers les organes lymphoïdes secondaires. L'augmentation parallèle de l'expression membranaire des molécules de co-stimulation lymphocytaire (CD80 et CD86) permet aux cellules dendritiques d'activer efficacement les lymphocytes T naïfs.

Par conséquent, seule la rencontre simultanée d'un antigène et d'un signal de maturation va provoquer le démarrage d'une réponse immunitaire, en tout cas pour ce qui est des lymphocytes T CD4 ${ }^{+}$. Ainsi, l'analyse de biologie cellulaire de la présentation antigénique au cours de la maturation a permis de comprendre les mécanismes fondamentaux qui permettent aux cellules dendritiques de débuter des réponses immunitaires, en déclenchant l'activation des lymphocytes $\mathrm{T} \mathrm{CD}^{+}$. La mise en route de réponses immunitaires cytotoxiques nécessite, de plus, l'activation des lymphocytes $\mathrm{T} \mathrm{CD}^{+}$.

\section{Présentation d'antigènes exogènes par les molécules de classe I du CMH}

Du point de vue des cellules dendritiques, deux classes d'antigènes induisant des réponses de type cytotoxique peuvent être distinguées. Tout d'abord, les antigènes produits par les cellules dendritiques elles- mêmes, comme les virus capables d'infecter les cellules dendritiques. Dans ce cas, la présentation des protéines virales synthétisées par les cellules dendritiques se fait par la voie conventionnelle. Dans cette voie de présentation, les antigènes sont digérés par le protéasome dans le cytosol et les peptides ainsi engendrés sont transférés par les transporteurs TAP1/2 dans la lumière du réticulum endoplasmique où ils s'associent aux molécules de classe I du CMH néosynthétisées [4]. De plus, les virus eux-mêmes induisent la maturation des cellules dendritiques, ce qui permet vraisemblablement un démarrage efficace des réponses immunitaires anti-virales (voir l'article de P. Benaroch et S. Le Gall, p.950 de ce numéro).

Le cas des antigènes déclenchant des réponses cytotoxiques, mais qui ne sont pas synthétisés par les cellules dendritiques elles-mêmes, est plus complexe. Les virus qui n'infectent pas les cellules présentatrices d'antigènes, mais d'autres tissus, ou les antigènes de tumeurs, représentent deux exemples de cette classe d'antigènes. Dans ces cas, les cellules dendritiques doivent acquérir des antigènes exogènes (provenant des tissus infectés ou des cellules tumorales) et présenter des peptides qui en dérivent sur les molécules de classe I du CMH [20] (figure 3). Ce processus, appelé présentation croisée (cross presentation), est en général, comme nous l'avons signalé plus haut, très inefficace in vitro. En effet, des concentrations très élevées d'antigènes solubles $(>1 \mathrm{mg} / \mathrm{ml})$, et donc non physiologiques, sont nécessaires pour obtenir une présentation antigénique sur les molécules de classe I du CMH.

Il existe cependant un certain nombre de situations lors desquelles la présentation efficace de peptides dérivés d'antigènes exogènes par les molécules de classe I du CMH est observée. L'équipe de K. Rock a montré que, lorsqu'un antigène est couplé à des billes synthétiques et internalisé par phagocytose, il peut être présenté efficacement à des lymphocytes $\mathrm{T}$ cytotoxiques [21]. Dans ce cas, la présentation est dépendante des transporteurs TAP1/2 et sensible à la lactacystine (un inhibiteur du protéasome), suggérant que les antigènes sont transportés 


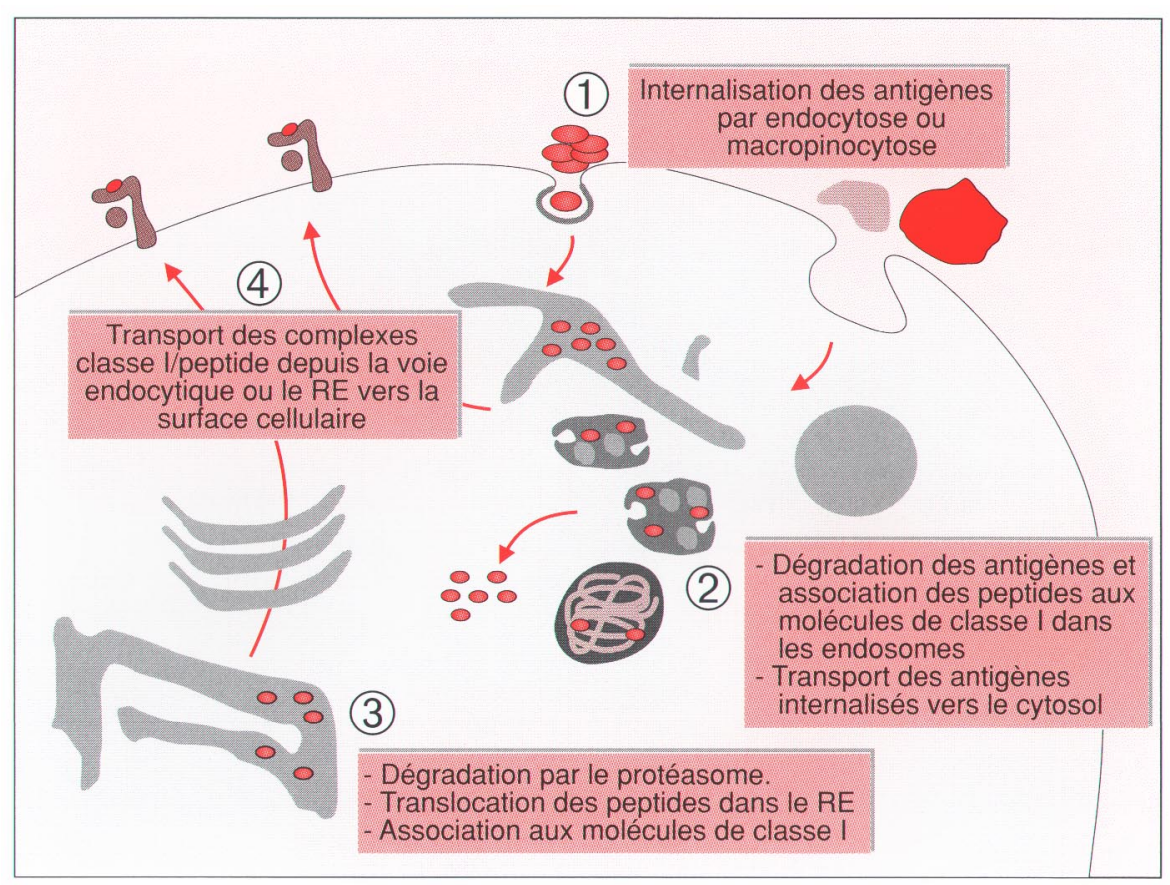

Figure 3. Voies de présentation d'antigènes exogènes par les molécules de classe I du CMH. Les antigènes internalisés peuvent être dégradés dans les endosomes et les peptides chargés sur des molécules de classe I du CMH, avant transport vers la surface cellulaire. Alternativement, les antigènes sont exportés vers le cytosol, où les antigènes sont dégradés par le protéasome, les peptides transloqués vers le réticulum endoplasmique par les transporteurs TAP et chargés sur les molécules de classe I du CMH néosynthétisées. $R E$ : réticulum endoplasmique.

depuis les phagosomes jusqu'au cytosol, avant de rejoindre la voie classique de présentation par les molécules de classe I du CMH. Cependant, les mécanismes par lesquels ces antigènes peuvent sortir des endosomes ou des phagosomes restent obscurs. L'équipe de C. Watts a montré que des ligands internalisés par macropinocytose étaient plus facilement transportés vers le cytosol, et a proposé que le transfert se fasse par rupture des macropinosomes ou des phagosomes [22]. Il n'existe cependant pas d'argument expérimental direct qui soutienne cette hypothèse.

Bien que la majorité des travaux réalisés à ce jour aient utilisé des macrophages, des études récentes montrent que dans les cellules dendritiques aussi, la macropinocytose et la phagocytose conduisent à une présentation efficace par les molécules de classe I du CMH [2325]. Cela est particulièrement clair dans le cas de la présentation d'antigènes exprimés par des bactéries. En effet, l'équipe de P. Ricciardi-Casta- gnoli [26] a montré qu'un antigène exogène recombinant exprimé par $S$. Gordoni était présenté de manière extrêmement efficace par des cellules dendritiques après infection. Cette voie de présentation est dépendante de TAP1/2, suggérant qu'ici aussi les antigènes sont transportés depuis les phagosomes vers le cytosol. Bien que ces résultats permettent de comprendre comment pourrait être mise en route une réponse contre des parasites intracellulaires, ils ne permettent pas d'expliquer les réponses contre des antigènes exprimés par des cellules différentes des cellules présentatrices d'antigènes.

Des études récentes ont décrit des voies différentes de cross presentation qui pourraient expliquer le transfert d'antigènes entre les cellules infectées ou tumorales et les cellules dendritiques. Tout d'abord, l'équipe de $\mathrm{N}$. Bhardwaj a montré que les cellules dendritiques peuvent phagocyter des cellules apoptotiques exprimant un antigène viral et présenter des peptides dérivés de cet antigène viral sur les molécules de classe I du CMH [27]. Des cellules nécrotiques exprimant le même antigène n'induisent pas de présentation par les molécules de classe I. De plus, cette fonction semble spécifique des cellules dendritiques, puisque des macrophages ayant phagocyté les mêmes cellules apoptotiques sont incapables de cross presentation.

Par ailleurs, nous avons montré récemment que lorsqu'un antigène associé à des anticorps spécifiques (complexes immuns) est internalisé par des RFc $\gamma$, des peptides dérivés de cet antigène sont présentés par les molécules de classe I du CMH [11]. Cette voie de présentation d'antigènes exogènes par les molécules de classe I du CMH est extrêmement efficace, puisqu'elle permet une activation de lymphocytes $\mathrm{T}$ cytotoxiques à des doses d'antigène 10000 fois inférieures aux doses nécessaires lorsque ce même antigène n'est pas complexé à des anticorps. Cette voie de présentation est spécifique des cellules dendritiques immatures, puisque ni les cellules dendritiques mûres, ni les macrophages, ni les lymphocytes B, capables d'internaliser des complexes immuns, ne présentent efficacement des antigènes après internalisation de ces complexes immuns. Comme la présentation d'antigènes particulaires après phagocytose, la voie dépendante des Rfc $\gamma$ nécessite les transporteurs TAP1/2 et un complexe protéasome fonctionnel (m/s 1993, $\left.n^{\circ} 11, p .1284\right)$, suggérant que là aussi, les antigènes doivent sortir des endosomes pour rejoindre la voie conventionnelle de présentation par les molécules de classe I du CMH. Puisque, comme nous l'avons indiqué plus haut, les $\mathrm{RF} \gamma \gamma$ provoquent aussi la maturation des cellules dendritiques, il est vraisemblable que la formation de complexes immuns puisse induire des réponses $\mathrm{T}$ cytotoxiques in vivo.

Ces différentes voies de présentation d'antigènes exogènes par les molécules de classe I jouent vraisemblablement des rôles plus ou moins importants dans le déclenchement ou le développement de réponses immunitaires particulières. Par exemple, on peut penser que l'ingestion de cellules apoptotiques joue un rôle prépondérant dans le démarrage de réponses cytotoxiques contre des virus, qui eux-mêmes induisent l'apoptose. Il est aussi probable, mais 
non encore démontré, que la présentation par les molécules de classe I d'antigènes provenant de cellules apoptotiques soit impliquée dans l'établissement de la tolérance immunitaire. En effet, des cellules de tous les tissus de l'organisme entrent en apoptose. On peut donc penser que l'ensemble des antigènes du soi puissent être, par ce moyen, ingérés par les cellules dendritiques et présentés aux lymphocytes $\mathrm{T}$ cytotoxiques par les molécules de classe I du CMH. Pour ces mêmes raisons, il est possible que l'apoptose soit impliquée dans le déclenchement de maladies auto-immunes. En effet, une augmentation d'entrée en apoptose au niveau d'un tissu donné, comme le pancréas dans le cas du diabète, pourrait provoquer la présentation d'antigènes spécifiques par les molécules de classe I du CMH des cellules dendritiques tissulaires, et aboutir à une rupture de tolérance, avec développement de lymphocytes $\mathrm{T}$ cytotoxiques dirigés contre des protéines du soi.

La présentation par les molécules de classe I du CMH après ingestion d'antigènes complexés à des anticorps spécifiques pourrait participer à la mise en route de réponses cytotoxiques secondaires. En effet, la plupart des réponses immunitaires cellulaires comportent aussi une composante humorale, qui aboutit à la production d'anticorps spécifiques. Nos résultats suggèrent l'existence d'interconnexions in vivo entre ces deux composantes des réponses immunes. Cette voie de présentation des complexes antigène-anticorps par les molécules de classe I du CMH pourrait aussi être impliquée dans la mise en route ou le développement de maladies auto-immunes où les complexes immuns participent à la pathogénie.

Enfin, quel que soit le mode d'acquisition des antigènes par les cellules dendritiques, il semble que la présentation de peptides dérivés des antigènes exogènes par les molécules de classe I du CMH puisse se faire par la voie conventionnelle ou directement dans les endosomes, probablement sur des molécules de classe I provenant de la surface cellulaire et capables de revenir à la surface cellulaire (figure 3). Il est vraisemblable que ces deux voies de présentation puissent être utilisées selon la production, ou non, des peptides dans l'environnement acide de la voie endocytique par des enzymes lysosomales. L'étude de ces deux voies de présentation, ainsi que des étapes de transport intracellulaire qu'elles nécessitent et des contraintes de spécificité qu'elles imposent, est maintenant nécessaire.

\section{Conclusions}

Bien que les mécanismes intimes aboutissant à la mise en route des réponses immunitaires restent en grande partie à éclaircir, de nombreuses études récentes suggèrent que les cellules dendritiques y jouent un rôle essentiel. Outre l'observation que les cellules dendritiques sont les seules cellules capables de stimuler des lymphocytes T naïs, leurs capacités de maturation fonctionnelle et de migration tissulaire, mettent les cellules dendritiques à une place unique parmi les différentes cellules présentatrices d'antigènes. Une somme remarquable d'efforts conjugués, par des dizaines d'équipes de par le monde, spécialisées dans une variété d'approches allant de la biologie moléculaire et cellulaire jusqu'à la médecine, en passant par l'immunologie, a permis en quelques années une accumulation extraordinaire de connaissances dans ce domaine. De nombreux essais précliniques chez l'animal, ainsi que les premiers essais cliniques utilisant les cellules dendritiques comme vecteur de vaccination (voir l'article de L. Zitvogel et F. Faure, p. 939 de ce numéro) ont donné des résultats très encourageants. Ces études laissent espérer que l'immunothérapie cellulaire utilisant des cellules dendritiques permettra de manipuler efficacemment les réponses immunitaires in vivo

\section{Remerciements}

Je remercie tous les membres de notre laboratoire pour les discussions et les idées qui ont nourri cet article, et Claire Hivroz pour l'avoir relu et corrigé.

\section{RÉFÉRENCES}

1. Germain RN. MHC-dependent antigen processing and peptide presentation: providing ligands for $\mathrm{T}$ lymphocyte activation. Cell 1994; 76 : 287-99.

2. Germain RN, Margulies DH. The biochemistry and cell biology of antigen processing and presentation. Annu Rev Immunol 1993; 11 : 403-50.

3. Carbone FR, Kurts C, Bennett SRM, Miller JFAP, Heath WR. Cross presentation: a general mechanism for CTL immunity and tolerance. Immunol Today 1998; 19: 368-73.

4. Bevan MJ. Cross priming for a secondary cytotoxic response to minor $\mathrm{H}$ antigens with $\mathrm{H}-2$ congenic cells which do not cross-react in the cytotoxic assay. J Exp Med 1976; 143 : 1283-8.

5. Hart DJ. Dendritic cells are unique leukocyte populations which control the primary immune response. Blood 1997; 90 : 3245-87.

6. Banchereau J, Steinman RM. Dendritic cells and the control of immunity. Nature 1998; 392: 245-52.

7. Steinman RM, Swanson J. The endocytic activity of dendritic cells. J Exp Med 1995; 182: 283-8.

8. Sallusto F, Cella M, Danieli C, Lanzavecchia A. Dendritic cells use macropinocytosis and the mannose receptor to concentrate macromolecules in the major histocompatibility complex class II compartment: downregulation by cytokines and bacterial products. J Exp Med 1995 ; 182: 389-400.

9. Ridge JP, Di Rosa F, Matzinger P. A conditioned dendritic cell can be a temporal bridge between a CD4 $4^{+}$T-helper and a T-killer cell. Nature 1998; 393: 474-8.

10. Schoenberger SP, Toes RE, Van der Voort EI, Offringa R, Melief CJ. T-cell help for cytotoxic $T$ lymphocytes is mediated by CD40-CD40L interactions. Nature 1998; 393: 480-3.

11. Regnault A, Lankar D, Lacabanne V, et $a l$. FcR-mediated induction of dendritic cell maturation and MHC class I-restricted antigen presentation after immune complex internalization. J Exp Med 1999; 189: 371-80.

12. Sallusto F, Lanzavecchia A. Efficient presentation of soluble antigen by cultured human dendritic cells is maintained by granulocyte/macrophage colony-stimulating factor plus interleukin 4 and downregulated by tumor necrosis factor alpha. I Exp Med $1994 ; 179$ : 1109-18.

13. Caux C, Liu YJ, Banchereau J. Recent advances in the study of dendritic cells and follicular dendritic cells. Immunol Today $1995 ; 16: 2-4$.

14. Inaba $\mathrm{K}$, Inaba $\mathrm{M}$, Romani $\mathrm{N}$, et al. Generation of large numbers of dendritic cells from mouse bone marrow cultures supplemented with granulocyte/macrophage colony-stimulating factor. J Exp Med 1992; 176: 1693-702. 


\section{RÉFÉRENCES}

15. Cella M, Engering A, Pinet V, Pieters J, Lanzavecchia A. Inflammatory stimuli induce accumulation of MHC class II complexes on dendritic cells. Nature 1997; 388: 782-6.

16. Saudrais C, Spehner D, De la Salle H, et al. Intracellular pathway for the generation of functional MHC class II peptide complexes in immature human dendritic cells. Immunol 1998; 160 : 2597-607.

17. Pierre P, Turley SJ, Gatti E, et al. Developmental regulation of MHC class II transport in mouse dendritic cells. Nature 1997 ; 388: 787-92.

18. Pierre P, Mellman I. Developmental regulation of invariant chain proteolysis controls MHC class II trafficking in mouse dendritic cells. Cell 1998; 93: 1135-45.

19. Cella M, Engering A, Pinet V, Pieters J, Lanzavecchia A. Inflammatory stimuli induce accumulation of MHC class II complexes on dendritic cells. Nature 1997; 388: 782-7.

20. Watts C. Capture and processing of exogenous antigens for presentation on MHC molecules. Annu Rev Immunol 1997; 15 : 821-50.

21. Rock KL, Gamble S, Rothstein L. Presentation of exogenous antigen with class I major histocompatibility complex molecules. Science 1990; 249 : 918-21.

22. Norbury CC, Hewlett LJ, Prescott AR, Shastri N, Watts C. Class I MHC presentation of exogenous soluble antigen via macropinocytosis in bone marrow macrophages. Immunity 1995; 3: 783-91.

23. Norbury CC, Chambers BJ, Prescott AR, Ljunggren HG, Watts C. Constitutive macropinocytosis allows TAP-dependent major histocompatibility complex class I presentation of exogenous soluble antigen by bone marrow-derived dendritic cells. Eur J Immunol 1997; 27: 280-8.

24. Shen Z, Reznikoff G, Dranoff G, Rock KL. Cloned dendritic cells can present exogenous antigens on both MHC class I and class II molecules. I Immunol 1997; 158 : 2723-30.

25. Mitchell DA, Nair SK, Gilboa E. Dendritic cell/macrophage precursors capture exogenous antigen for MHC class I presentation by dendritic cells. Eur I Immunol 1998 ; 28 : 1923-33.

26. Rescigno M, Citterio S, Thery C, et al. Bacteria-induced neo-biosynthesis, stabilization, and surface expression of functional class I molecules in mouse dendritic cells. Proc Natl Acad Sci USA 1998; 95 : 5229-34.

27. Albert ML, Sauter B, Bhardwaj N. Dendritic cells acquire antigen from apoptotic cells and induce class I-restricted CTLs.

\section{Summary}

Antigen presentation by dendritic cells

The stimulation of $\mathrm{T}$ lymphocytes requires the presentation of proteolytic antigen-derived peptides by major histocompatibility complex (MHC) molecules. All cells in the organism express MHC class I molecules, which present peptides derived from endogenous antigens to cytotoxic T cells. In contrast, only a few cell types, mainly from hematopoietic origin, express MHC class II molecules and are capable of stimulating helper $\mathrm{CD} 4^{+}$ $\mathrm{T}$ lymphocytes. One of these cell types, dendritic cells (DC), have the unique capacity to stimulate naive $\mathrm{T}$ lymphocytes and initiating primary and secondary immune responses. This unique role of DCs relies on three specific attributes of this particular antigen presenting cells. First, their capacity to undergo a series of phenotypical and functional changes (maturation) in response to inflammatory signals. These modifications cause the migration of DCs from peripherical tissues and mucosa (where DCs reside in their immature state) towards secondary lymphoid organs, where DCs may stimulate resting $\mathrm{T}$ lymphocytes. Second, a tightly regulated MHC class II antigen presentation capacity. Only immature DCs ingest and process antigens, whereas only the mature ones present peptides to $\mathrm{CD}^{+} \mathrm{T}$ lymphocytes. Finally, a unique ability to present peptides from exogenous antigens, internalized from the extracellular milieu, on MHC class I molecules. Together, these specific attributes confer to DCs a central role in the initiation of both humoral and cellular immune responses.

\section{TIRÉS À PART}

S. Amigorena. 